\title{
Avaliação psicométrica em português do indicador de dor crônica de Helsinki em cães com sinais crônicos de osteoartrite
}

\author{
[Psychometric testing in Portuguese of Helsinki's chronic pain index for dogs with chronic \\ signs of osteoarthritis] \\ L.M. Matsubara ${ }^{1}$, S.P.L. Luna ${ }^{1}$ L.R. Teixeira ${ }^{1}$, M.S. Castilho ${ }^{1}$, A.H. Björkman ${ }^{2}$, \\ H.S. Oliveira ${ }^{1}$, L.F.C. Anunciação ${ }^{3}$ \\ ${ }^{1}$ Universidade Estadual Paulista - Botucatu, SP \\ ${ }^{2}$ University of Helsinki - Helsinki, Finland \\ ${ }^{3}$ Pontifícia Universidade Católica do Rio de Janeiro - Rio de Janeiro, RJ
}

\begin{abstract}
RESUMO
Objetivou-se determinar a validade, a confiabilidade e a sensibilidade do indicador de dor crônica de Helsinki (IDCH) em português, além de estabelecer correlação entre a plataforma de pressão em cães com displasia coxofemoral (DCF). O questionário foi preenchido por tutores de 40 animais com DCF e por 16 tutores de cães saudáveis. Os animais com DCF foram tratados com carprofeno $4,4 \mathrm{mg} / \mathrm{kg}$ (GT= 21) ou placebo $(\mathrm{GP}=19)$, administrados uma vez ao dia, durante quatro semanas. A avaliação consistiu no preenchimento do questionário (IDCH), na escala analógica visual de dor (EAVdor) e na escala analógica visual de locomoção (EAVloc) com duas semanas (A1) e imediatamente antes do tratamento (A2), duas (S2) e quatro (S4) semanas de tratamento e após duas semanas do término do tratamento (S6). A consistência interna dos dados foi considerada excelente (alfa de Cronbach $=0,89$ ). Houve correlação moderada entre o IDCH e a EAVdor e boa entre o IDCH e a EAVloc, porém não houve diferença entre o grupo tratado com carprofeno e placebo no IDCH. Não houve correlação entre os dados da plataforma de pressão e o IDCH. O questionário apresentou validade de constructo, critério e fidedignidade e pode-se empregá-lo em países de língua portuguesa em cães com osteoartrite.
\end{abstract}

Palavras-chave: cães, dor crônica, osteoartrite, estudos de validação

\begin{abstract}
We aimed to determine validity, reliability, and sensitivity of Helsinki's chronic pain index (HCPI) and stablish a correlation between HCPI in dogs with hip dysplasia (HD) using pressure sensitive walkway. Forty-owners of dogs with HD and 16 owners of health dogs filled a questionnaire. Dogs with HD were treated with carprofen $4.4 \mathrm{mg} / \mathrm{kg}(G T \mathrm{n}=21)$ or with placebo $(G P \mathrm{n}=19)$, both were administered once a day for 4 weeks. Evaluation was performed by the owners using the questionnaire (HCPI), the Visual Analogue Scale for pain (VASpain) and the VAS for locomotion (VASloc). The evaluation was performed 2 weeks before the treatment began (A1), immediately after treatment (A2), two (S2), four (S4) and two weeks after the end of treatment (S6) and the lameness was evaluated by pressure sensitive walkway. The internal consistency of the data was considered excellent (Cronbach' $\alpha$ coefficient $=0.89$ ). There was a moderate correlation between the HCPI and VASpain. For VASloc the correlation was good. However, there was no difference between treatments, indicating low sensibility. No correlation was observed between pressure sensitive walkway and HCPI. We concluded that the questionnaire has construct and criterion validity, reliability and can be applied in dogs with osteoarthritis in Portuguese-speaking countries.
\end{abstract}

Keywords: dogs, chronic pain, osteoarthritis, validation studies

Recebido em 14 de março de 2017

Aceito em 25 de janeiro de 2018

E-mail: lidia27_matsubara@yahoo.com.br 


\section{Matsubara et al.}

\section{INTRODUÇÃO}

A displasia coxofemoral (DCF) é uma alteração hereditária que envolve a ausência de conformidade entre a cabeça do fêmur e o acetábulo e causa osteoartrite (OA) (Lust, 1997). O diagnóstico é feito pelo exame radiográfico, porém não há uma correlação direta entre o grau de dor e a gravidade das alterações radiográficas (Brass, 1989).

O reconhecimento da dor é fundamental para tratá-la. O desafio em mensurar a dor na medicina veterinária deve-se à ausência de métodos validados e com confiabilidade testada, que mensurem a dor em pacientes que não possuem a capacidade de se expressar verbalmente (Sharkey, 2013). Uma das escalas validadas em inglês para avaliar a dor crônica em cães com osteoartrite é a escala de Helsinque (Hielm-Björkman et al., 2009), que consiste em 11 questões respondidas pelos tutores. Essa ferramenta foi validada para avaliar a dor crônica em cães com osteoartrite, que apresentavam escores significativamente maiores que os saudáveis. Alem disso, ao serem tratados com anti-inflamatório, observava-se redução dos escores dor frente ao tratamento.

Atualmente a avaliação da dor nesses pacientes é feita de maneira subjetiva pelos tutores, que descrevem alterações no comportamento do animal, como diminuição na atividade física, do apetite, aumento da agressividade e claudicação (Wiseman-Orr et al., 2001), porém não é possível quantificar a dor ou a necessidade de tratamento sem o emprego de uma escala validada. As alterações comportamentais associadas com a dor crônica podem ser graduais ou súbitas e somente são percebidas por pessoas muito próximas e que convivem diariamente com os animais, como no caso dos tutores e, por isso, o questionário é preenchido por eles, e não por médicos veterinários, já que, de acordo com vários estudos, não há boa precisão por parte dos veterinários na quantificação da dor crônica, enquanto os tutores são as pessoas mais indicadas para avaliá-la (Hielm-Björkman et al., 2003; Sharkey, 2013).

A validade é a eficiência com que uma escala mede o que se investiga e divide-se em validade de conteúdo, constructo e critério (Morton et al., 2005). A validade de conteúdo se refere à adequação de que todos os itens incluídos na escala se referem aos aspectos medidos. A validade de critério é a eficiência de mensuração da escala comparada a um padrão bem reconhecido (Morton et al., 2005), e a validade de constructo, evidência mais forte da validade, é determinada quando o instrumento fornece as respostas que preenchem a hipótese na qual se desenvolveu o questionário (Wiseman-Orr et al., 2006).

A confiabilidade é a garantia de a medida produzir o mesmo escore toda vez que for usada (Hielm-Björkman et al., 2009). A consistência interna é o primeiro teste de confiabilidade e determina como os itens que refletem o mesmo constructo fornecem resultados similares, pelo uso do alfa de Cronbach. Obtém-se a confiabilidade intraobservador ou repetibilidade ou estabilidade quando os mesmos indivíduos aplicam o teste duas vezes em momentos diferentes (Hielm-Björkman et al., 2009).

Com base na justificativa de que há diferenças importantes ao se adaptarem escalas de uma língua para outra (Sperber, 2004) e diante da necessidade de validação semântica e linguística de escalas em português para avaliar dor crônica em cães, os objetivos deste trabalho foram validar em português o IDCH durante a aplicação clínica do instrumento em cães portadores de DCF e verificar se há correlação entre o questionário e a plataforma de pressão.

\section{MATERIAL E MÉTODOS}

Parte dos dados deste estudo foram utilizados em estudo prévio para comparar a eficácia da acupuntura versus o carprofeno em animais com dor crônica (Teixeira et al., 2016).

O estudo foi aprovado pela Comissão de Ética para Uso de Animais da instituição, sob o protocolo de número 204/2010, e os tutores assinaram o Termo de Consentimento.

O questionário indicador de dor crônica de Helsinki (IDCH) (Hielm-Björkman et al., 2009) foi traduzido do finlandês para o português por dois tradutores fluentes nas duas línguas. As duas versões foram revisadas, comparadas e transformadas em uma única versão. A nova versão foi traduzida novamente para o finlandês pelas mesmas pessoas. Os autores do artigo 
compararam e revisaram as versões para assegurar a adequação da tradução.

Quarenta cães com sinais clínicos e radiográficos de DCF e 16 cães saudáveis foram avaliados clínica e radiograficamente. Para inclusão no estudo, além do laudo radiográfico de DCF uni ou bilateral, de grau moderado a grave, os tutores deveriam relatar pelo menos dois dos quatro sinais clínicos a seguir: dificuldade 1) em deitar ou levantar da posição deitada, 2) de pular ou recusa em pular, 3) de subir ou descer escadas ou 4) claudicação. Adicionalmente, os cães deveriam apresentar dor por mais de três meses, de acordo com informação dos tutores. Excluíram-se os cães sob tratamento com analgésicos e condroprotetores por, no mínimo, quatro semanas antes do início do estudo.

Após jejum alimentar de 12 horas e hídrico de duas horas, aplicou-se morfina (Dimorf, Cristália, Brasil), na dose de 0,5mg/kg, IM, seguida de propofol (Propovan, Cristália, Brasil), pela via IV, com dose que permitisse realizar as avaliações radiográficas nas projeções ventrodorsal e laterolateral do quadril e coluna cervical, torácica, lombar e sacral e projeções mediolateral e craniocaudal do joelho e cotovelo. As avaliações da articulação coxofemoral seguiram os padrões da Orthopedic Foundation for Animals (OFA) (Flückiger, 2007).

Incluíram-se apenas animais que apresentavam normalidade de hemograma, ureia, creatinina, ALT, FA, proteína total e albumina antes do início do tratamento (A1). Distribuíram-se os animais por sorteio aleatório (Research Randomizer, versão 4.0, Geoffrey C. Urbaniak and Scott Plous, EUA) em dois grupos: GT - 21 cães com displasia tratados com $4,4 \mathrm{mg} / \mathrm{kg}$ de carprofeno (Carproflan, Agener União, Brasil) SID, por via oral, ou GP - 19 cães com displasia tratados com $1 \mathrm{mg} / \mathrm{kg}$ de lactose (placebo) SID, com cápsulas idênticas às do carprofeno. Realizou-se o tratamento por quatro semanas. Os tutores e pesquisadores responsáveis pela pesquisa eram encobertos, e os tutores tinham conhecimento de que havia um grupo tratado e um placebo. No caso de piora dos sinais clínicos e de episódios de dor, os tutores, caso julgassem necessário, eram instruídos a administrarem $4 \mathrm{mg} / \mathrm{kg}$ de tramadol, via oral, TID, e comunicavam os pesquisadores. Os animais eram resgatados da mesma forma, caso, nas consultas, a pontuação total do questionário atingisse $\mathrm{o}$ valor de 30\% (Brondani et al., 2013).

O questionário de Hielm-Björkman et al. (2009) foi aplicado em cães com DCF, e os tutores preencheram-no em cada momento de avaliação. As respostas foram referentes ao estado do animal na semana anterior. A somatória das 11 questões fornece o valor máximo de 44 . Conferem-se os escores 0 e 1 de cada questão para cães sem dor e os escores de 2 a 4 para aqueles com dor crônica. Pontuações menores que 11 se associam à ausência de dor e maiores que 12 à dor crônica (Anexo 1).

Os tutores responderam ao questionário duas semanas (A1) e imediatamente antes (A2) do início do tratamento, duas (S2) e quatro (S4) semanas após o início do tratamento e duas semanas após o término do tratamento (S6). Os tutores também preencheram a escala analógica visual de dor (EAVdor) e de locomoção (EAVloc). Para tal, eram orientados a assinalar um x sobre uma linha de $10 \mathrm{~cm}$, com a finalidade de avaliar as suas percepções sobre a dor e a locomoção dos seus cães, respectivamente. O extremo esquerdo da linha indicava ausência de dor ou de dificuldade para locomoção, e o extremo direito da linha representava a pior dor possível ou a mais grave dificuldade de locomoção. Na segunda e quarta semanas de tratamento, os tutores foram orientados a preencherem sobre a qualidade de vida $(\mathrm{QV})$ do animal, em que 1 era muito melhor; 2 melhor; 3 igual; 4 pior e 5 muito pior, em relação a antes do início do tratamento.

Avaliou-se a claudicação pela análise cinética, por meio de uma plataforma de pressão (Walkway TM High Resolution HRV4, Tekscan, EUA) montada ao nível do solo, com uma interface conectada a um computador provido de um programa de processamento de dados (Walkway, version 7.0, Tekscan, EUA). Os animais foram conduzidos sobre a plataforma, ao passo, a uma velocidade constante de 0,9 a $1,1 \mathrm{~m} / \mathrm{s}$ e aceleração entre $-0,2$ e $0,2 \mathrm{~m} / \mathrm{s}^{2}$. Os dados coletados foram o índice de simetria do pico de força vertical (ISPFV) e o índice de simetria do impulso vertical (ISIV) dos membros pélvicos.

Os cães saudáveis $(n=16)$ foram avaliados em um único momento, para se descartar dor crônica 
segundo os tutores, e a radiografia foi realizada para se descartarem sinais radiográficos de DCF.

$\mathrm{Na}$ avaliação das propriedades psicométricas do instrumento em A1 e A2, utilizaram-se os animais de GP e GT. Antes de se avaliarem as técnicas psicométricas relacionadas à fatoração, testou-se a hipótese da adequação da amostra pela medida Kaiser-Meyer-Olkin (KMO) e da esfericidade dos dados pelo teste de Bartlett. Para avaliar a validade de constructo, a análise de componentes principais (ACP) foi realizada sem nenhuma restrição. A análise dos resultados permitiu realizar, novamente, a ACP, estipulando-se uma solução unidimensional com rotação Varimax.

Em seguida, a validade de critério foi avaliada pela correlação de Spearman entre os resultados do IDCH e da escala de QV, EAVdor e EAVloc. $\mathrm{Na}$ sequência, analisou-se a consistência interna do instrumento considerando o Alfa de Cronbach $(\alpha)$. Para se analisar a diferença de respostas entre o tratamento farmacológico e o placebo, verificou-se a aleatorização dos grupos, considerando-se o nível de medida de cada variável a partir do qui-quadrado, da ANOVA e do teste $\mathrm{T}$ independente. Os resultados do IDCH foram analisados pelo teste de Mann-Whitney e pela ANOVA de medidas repetidas. As comparações entre o ISPFV e o ISIV foram analisadas pelo Mann-Whitney e correlacionadas com a EAVdor e a EAVloc.

Aceitaram-se valores > 0,6 para a medida KMO, qui-quadrado significativo para o Bartlett, carga fatorial > 0,4 para cada item, variabilidade explicada $>0,5$ no resultado dimensional da ACP e significância de 0,05 . As análises foram realizadas no programa SPSS V.22.

\section{RESULTADOS}

Os cães sadios eram de médio ou grande porte, com idade variando de um ano e meio a oito anos, pertencentes a diversas raças. Não houve diferença entre os grupos para a massa corpórea
GT $(34 \mathrm{~kg} \pm 8,4), \mathrm{GP}(32 \mathrm{~kg} \pm 8,8)$ e GC $(29 \mathrm{~kg} \pm 8,6)$, porém os animais do GC $(2,9$ anos $\pm 1,9)$ apresentaram idade inferior aos animais com DCF: GT $(6,3 \pm 4,2)$ e GP $(6,1 \pm 4,4$ anos). Não houve diferença entre o grau de escolaridade dos tutores entre os grupos.

Em relação ao grau de displasia, não houve diferença entre os grupos GT e GP. De 44 animais displásicos avaliados, excluíram-se quatro, todos machos. Um foi pela piora dos sinais neurológicos, outro pela administração concomitante de medicamentos e dois pela não administração do medicamento conforme recomendações, já que apresentaram efeitos adversos (um animal do GT perdeu o apetite e outro apresentou vômito), sendo tratados com omeprazol $(1 \mathrm{mg} / \mathrm{kg}$, via oral, SID) e tendo apresentado boa recuperação.

De 21 animais do GT, apenas três $(14,3 \%)$ receberam resgate analgésico com tramadol. Em um animal, administrou-se o tramadol por seis dias, entre S2 e S4; em outro, por 10 dias, entre A2 e S2; e, no último, entre S2 e S4. Nenhum animal de GP necessitou resgate.

A medida Kaiser-Meyer-Olkin foi de 0,79, o teste de esfericidade de Bartlett apresentou $\chi^{2}(55)$ $=237,16, \mathrm{P}<0,001$, o que indica que os dados foram adequados à análise fatorial. A ACP e os valores de correlação interitem se encontram na Tab. 1.

A ACP em A1 e A2 resultou na extração de três componentes com autovalor $>1$. Considerou-se o instrumento unidimensional, pois a carga fatorial dos itens teve valor $>0,4$, a distância entre as duas primeiras dimensões é três vezes maior do que entre a segunda e a terceira, e a variância explicada é superior a $50 \%$ (Fig. 1). Este componente contém nove itens fortes, com cargas fatoriais semelhantes em A1 e A2 e dois itens com dispersão, que são: o item 1 (ânimo), com carga fatorial de 0,30 a 0,54 , e o item 3 (vocalização), de 0,30 a 0,57 (Fig. 2). 
Avaliação psicométrica...

Tabela 1. Valores do indicador de dor crônica de Helsinki (IDCH) (média \pm DP) para o total de itens e para os itens individuais com a análise dos componentes principais (ACP) e correlação item-total e valores do alfa de Cronbach $(n=56)$

\begin{tabular}{cccccccc}
$\begin{array}{c}\text { IDCH } \\
\text { itens }\end{array}$ & A1 & A2 & A1-A2 & $\begin{array}{c}\text { Carga } \\
\text { fatorial }\end{array}$ & $\begin{array}{c}\text { Comunalidades } \\
\left(\mathrm{h}^{2}\right)\end{array}$ & $\begin{array}{c}\text { Correlação } \\
\text { item-total }\end{array}$ & $\alpha$ \\
\hline Total & $12,73 \pm 8,75$ & $16,70 \pm 6,67$ & $-3,97$ & NA & NA & NA & 0,90 \\
1 & $0,79 \pm 0,65$ & $1,21 \pm 0,77$ & $-0,42$ & 0,55 & 0,30 & 0,45 & 0,90 \\
2 & $0,82 \pm 0,90$ & $1,21 \pm 0,86$ & $-0,38$ & 0,69 & 0,48 & 0,62 & 0,89 \\
3 & $0,73 \pm 0,88$ & $0,95 \pm 0,83$ & $-0,22$ & 0,30 & 0,09 & 0,25 & 0,91 \\
4 & $1,04 \pm 0,76$ & $1,36 \pm 0,71$ & $-0,32$ & 0,77 & 0,59 & 0,70 & 0,88 \\
5 & $1,20 \pm 0,98$ & $1,56 \pm 0,91$ & $-0,37$ & 0,77 & 0,60 & 0,69 & 0,88 \\
6 & $1,30 \pm 1,13$ & $1,62 \pm 1,09$ & $-0,31$ & 0,83 & 0,68 & 0,75 & 0,88 \\
7 & $1,45 \pm 1,28$ & $1,97 \pm 1,06$ & $-0,53$ & 0,82 & 0,67 & 0,78 & 0,88 \\
8 & $0,91 \pm 0,86$ & $1,31 \pm 0,77$ & $-0,40$ & 0,62 & 0,39 & 0,54 & 0,89 \\
9 & $1,46 \pm 1,14$ & $1,85 \pm 0,87$ & $-0,38$ & 0,78 & 0,60 & 0,70 & 0,88 \\
10 & $1,30 \pm 0,95$ & $1,62 \pm 0,88$ & $-0,31$ & 0,77 & 0,59 & 0,69 & 0,88 \\
11 & $1,55 \pm 1,04$ & $1,97 \pm 0,81$ & $-0,42$ & 0,75 & 0,56 & 0,68 & 0,88 \\
\hline
\end{tabular}

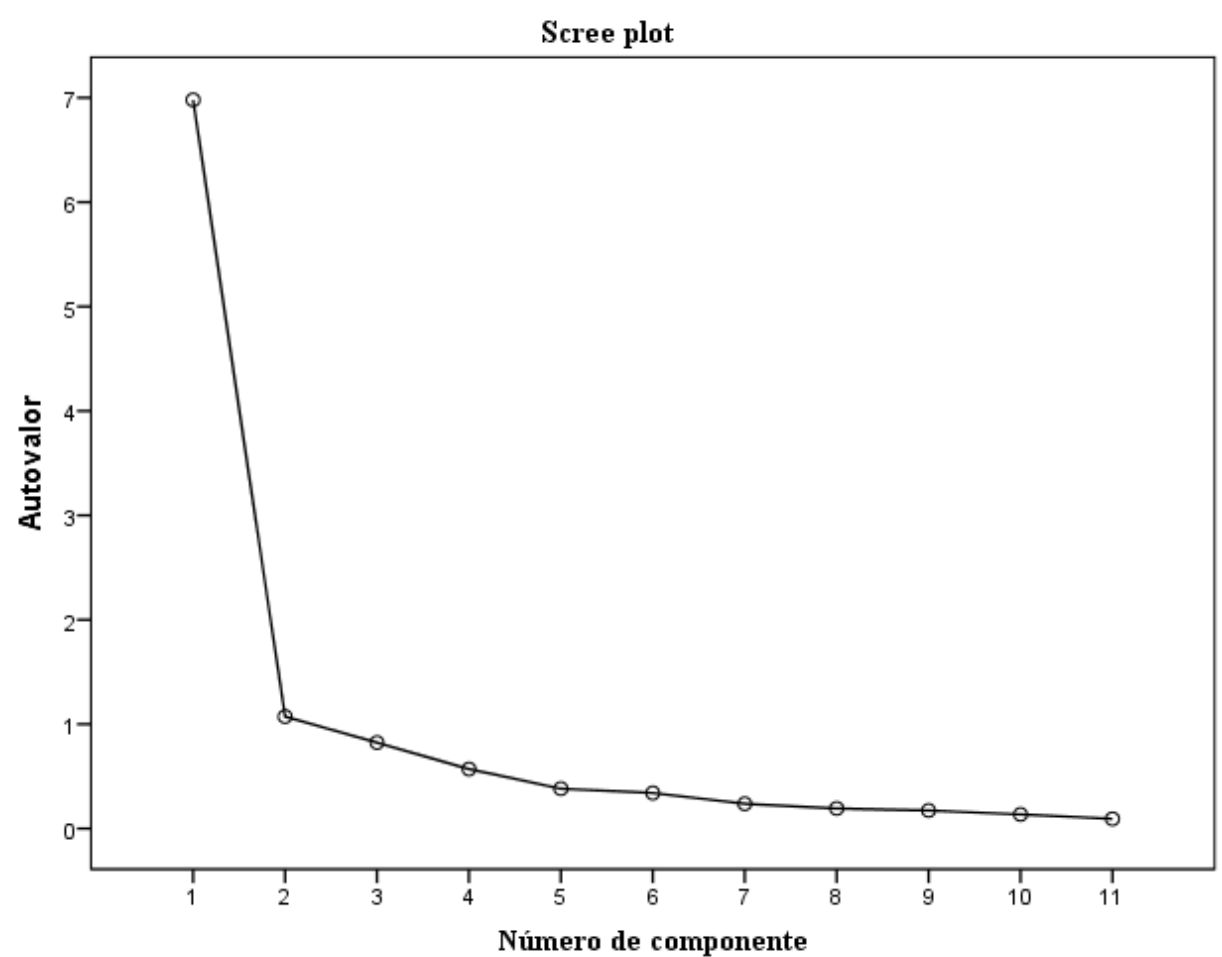

Figura 1. Gráfico de autovalores do indicador de dor crônica de Helsinki (IDCH) em A1. A curva revela que o IDCH é explicado como um índice de um componente único (dor crônica), incluindo todos os 11 itens $(n=56)$. 


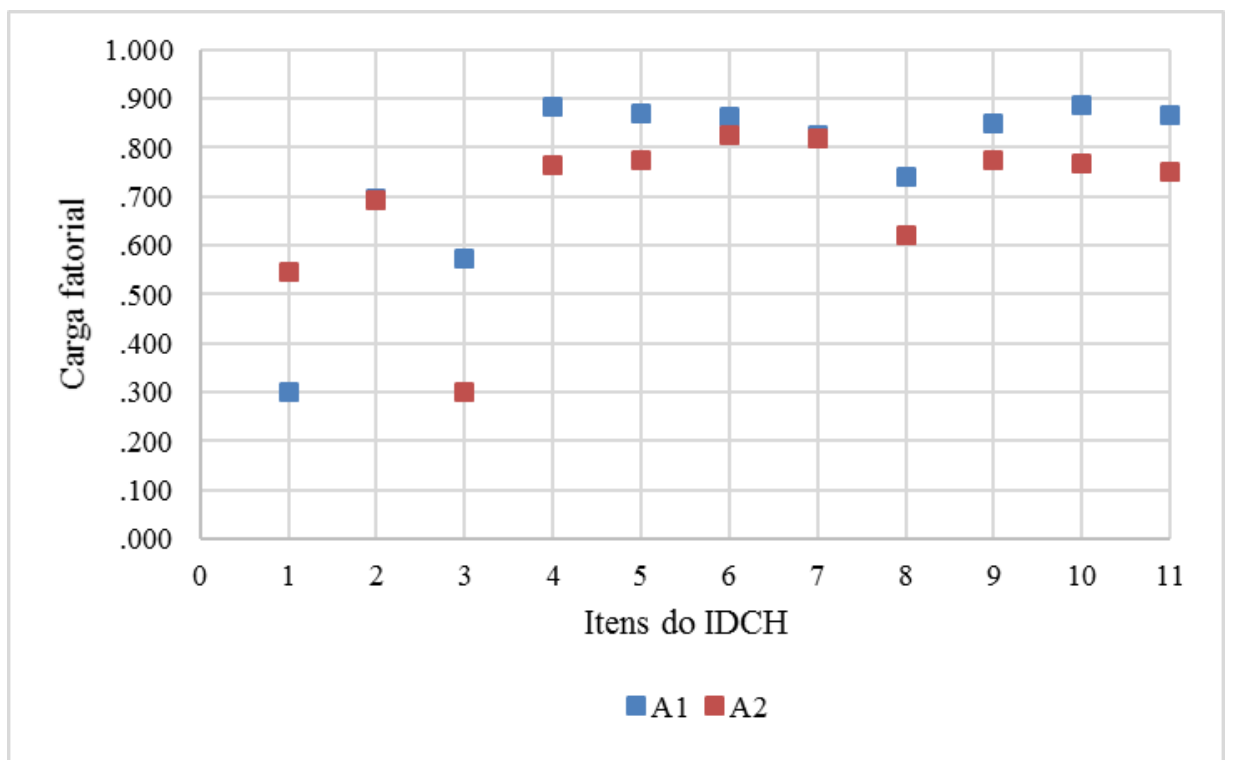

Figura 2. Cargas fatoriais dos 11 itens nos momentos basais (A1 e A2). O resultado do indicador de dor crônica de Helsinki (IDCH) é explicado por diferentes aspectos associados à dor crônica. A proximidade dos valores nos dois pontos indica que os tutores responderam de maneira similar após intervalo de duas semanas $(n=56)$.

As alterações no IDCH correlacionaram com alterações na EAVdor, EAVloc e QV. A comparação entre as medidas, feita de forma concorrente, demonstrou uma correlação razoável entre o IDCH (A1) e a QV; a correlação do IDCH e das EAVs foi proporcional. Em A1, a correlação entre o IDCH e a EAVloc foi de 0,59 (moderada) e com a EAVdor foi de 0,79 (boa). No A2, para a EAVloc, foi de 0,56 (moderada), e para a EAVdor foi de 0,63 (boa). No S2, a correlação entre o IDCH e a EAVloc foi de 0,55, enquanto para a EAVdor foi de 0,61 (ambas moderadas).

Como se aceitou a unidimensionalidade do instrumento, a análise da fidedignidade contou com o estimador $\alpha$ de Cronbach, usado para correlacionar cada um dos itens e o total do instrumento. Como se realizaram duas avaliações basais (A1 e A2), aplicou-se o teste-reteste, que atingiu o $\alpha$ de Cronbach 0,89. Se algum dos itens fosse excluído, o valor do $\alpha$ de Cronbach seria de 0,88 a 0,91 .

A confiabilidade intraobservadores, concordância ou repetibilidade foi avaliada ao se comparar o A1 e o A2, no GT e GP pela correlação de Pearson, que foi de 0,84. Não houve diferença entre os momentos ao longo do tempo em cada grupo ou entre os grupos em cada momento. Não houve diferença nos valores da mediana e semiamplitude total do ISPFV e ISIV entre os cães saudáveis $(0,77 \pm 0,99$ e $0,83 \pm 0,69)$ e os displásicos no GT $(1,01 \pm 1,35 ; 1,21 \pm 2,11)$ e GP $(1,43 \pm 1,36 ; 2,14 \pm 0,90)$, em A1. Não houve diferença entre os momentos e os grupos $(\mathrm{P}>0,05)$.

Os escores da EAVdor e EAVloc (mediana \pm semiamplitude total) no GC foram menores $(0 \pm 0)$ que nos demais grupos em A1 $(\mathrm{P}<0,05)$. No GT, houve redução da EAVdor em S2 $(2,0 \pm 1,8)$ e S4 $(2,0 \pm 1,1)$, mas não em S6 $(2,4 \pm 2,3)$ em relação a A1 $(4,5 \pm 1,2)$ e na EAVloc apenas em S4 $(2,5 \pm 1,6)$, mas não em S2 $(2,8 \pm 1,8)$ e S6 $(3,0 \pm 1,8)$ em relação a A1 $(4,9 \pm 1,7)$. Em GC, não houve diferença ao longo do tempo nem para a EAVdor em A1 $(4,2 \pm 2,0)$; S2 $(2,5 \pm 1,8) ; \quad$ S4 $(2,2 \pm 1,9)$ e S6 $(1,7 \pm 1,7)$ ( $\mathrm{P}>0,05)$, nem para a EAVloc em A1 $(3,5 \pm 1,7)$; S2 $(2,5 \pm 1,7) ; \quad S 4 \quad(1,6 \pm 1,4)$ e S6 $(1,5 \pm 2,0)$ $(\mathrm{P}>0,05)$.

Houve redução do escore do IDCH (mediana \pm semiamplitude total) no GT, em S4 $(12 \pm 4,5)$ em relação a A1 $(17 \pm 2,6)$ e A2 $(18 \pm 4,2)$, e no GP, redução em S4 $(15 \pm 3,5)$ e S6 $(13 \pm 4,2)$ em relação a A2 $(18 \pm 4,2)$. Os escores do IDCH no $\mathrm{GC}$, em A1 $(0 \pm 2,7)$ foram menores que os obtidos em GT $(17 \pm 2,6)$ e GP $(15 \pm 5,2)(\mathrm{P}<0,05)$. 


\section{DISCUSSÃO}

Para se utilizar um instrumento que mensure a dor, deve-se submetê-lo a testes estatísticos que determinem a validade e a confiabilidade (Hielm-Björkman et al., 2009). Seguiram-se alguns passos propostos anteriormente (Guillemin, 1995) para adaptação e validação transcultural de instrumentos de medida, como a tradução, o pré-teste e a retrotradução. Não se realizaram os passos de avaliação por comitê de juízes e determinação dos pesos dos escores no contexto cultural, por se tratar de um questionário com índices de validade e confiabilidade já reconhecidos e, portanto, julgou-se suficiente apenas validar a tradução.

O IDCH é um instrumento válido e confiável para uso em português e unidimensional, ou seja, avalia a dor crônica, agrupando-se os 11 itens. Diversos fatores levam a essa conclusão: o primeiro componente reteve $50,4 \%$ da variância, e os outros 10 componentes de 0,9 a $12 \%$ em um padrão linear (Fig. 1), o que indica que somente um componente poderia ser retido, de acordo com a curva do gráfico do autovalor. $\mathrm{O}$ item 1 (ânimo) e o item 3 (vocalização) apresentaram carga fatorial menor que os outros itens, mas ainda permanecem consistentes, já que se comportaram do mesmo modo em A1 e A2 (Fig. 2). Como se está apenas validando o IDCH em português, não se poderiam excluir esses itens.

Pela validade de critério, a correlação entre o IDCH e a QV, a EAVdor e a EAVloc foi razoável e de moderada a boa, respectivamente. Devido à ausência de um padrão-ouro (Morton et al., 2005) para compará-lo com o IDCH, empregaram-se a EAVdor e a EAVloc, pois, embora não validadas, são amplamente utilizadas (Hielm-Björkman et al., 2011). Isto se confirma pela redução dos valores da EAVdor (4,5 para $2,0)$ e da EAVloc $(4,9$ para 2,5), do A1 para o S4, no GT, o que indica boa sensibilidade dessa escala em detectar melhora dos animais submetidos ao tratamento com anti-inflamatório. Porém, as escalas analógicas são demasiadamente simples e unidimensionais, e não possuem validade de conteúdo (HielmBjörkman et al., 2011). Já o IDCH é um questionário multidimensional, que avalia alterações na locomoção, no comportamento e na atitude dos animais, o qual pode ser confiável e fornecer resultados semelhantes, mesmo quando utilizado por pessoas inexperientes (HielmBjörkman et al., 2011). A ausência de diferença entre GT e GP, no IDCH, provavelmente se deve ao número reduzido de animais necessários para detectar diferença estatística e à escolha da displasia coxofemoral como critério de inclusão. Esta afirmação é corroborada pelo fato de também não se observar diferença tanto para a EAVdor como para a EAVloc entre o grupo placebo e o tratado.

A pobre correlação entre as variáveis objetivas de claudicação (ISIV e ISPFV) e o IDCH já foi relatada previamente para a plataforma de força (Walton et al., 2013). Esperava-se uma correlação entre a plataforma de pressão e o IDCH, que não ocorreu, pois a análise fatorial não extraiu um componente que descrevesse a claudicação baseado na carga fatorial dos itens (Walton et al., 2013). Aparentemente os tutores têm mais facilidade em avaliar a dor do seu animal apenas se o animal claudicar. No presente estudo, a falha da avaliação cinética, tanto em identificar animais com claudicação, como em apontar melhoras dela após o tratamento, foi provavelmente porque a claudicação é alta e, por isso, é visível somente no trote e em pisos duros (Voss et al., 2010). A displasia coxofemoral é uma alteração que promove alterações complexas na locomoção. É difícil padronizar se o comprometimento é uni ou bilateral e se sua compensação na área de contato entre o membro acometido e o contralateral, o que pode justificar as razões pelas quais os índices de simetria ISIV e ISPFV não demonstraram alterações em cães saudáveis e displásicos, como relatado anteriormente (Oosterlinck et al., 2011; Teixeira et al., 2016).

O carprofeno foi escolhido como controle positivo, pois é amplamente utilizado para tratar OA (Vasseur et al., 1995; Lipscomb et al., 2002) e apresenta efeito comparável a outros antiinflamatórios (Edamura et al., 2012; Reymond et al., 2012). Entretanto, o efeito do carprofeno pode ser limitado em animais com DCF grave.

Talvez a inclusão de outros tratamentos farmacológicos (Johnston et al., 2008) ou não farmacológicos (Rychel, 2010) ao carprofeno pudesse detectar com mais propriedade alguma melhora na claudicação da avaliação cinética, pela necessidade de se lançar mão de fármacos 
adjuvantes e tratamento não farmacológico para a dor crônica.

Há três formas de se avaliar a confiabilidade: a consistência interna, a repetibilidade e a sensibilidade (Hielm-Björkman et al., 2009). A ausência de diferença entre o GT e o GP no IDCH, já que se esperaria que no GT os escores se reduzissem após o tratamento, pode ser atribuída à baixa sensibilidade da escala e/ou ao efeito placebo e/ou à ineficácia do carprofeno na DCF; este último já discutido anteriormente. A sensibilidade da escala já foi aprovada em estudo anterior (Hielm-Björkman et al., 2009), utilizando-se um número de animais inclusive inferior ao do presente estudo (17 versus 19/21). Os menores valores do IDCH nos cães saudáveis (GC) em relação ao GT e ao GP indicam que a escala apresenta certa sensibilidade. Aparentemente, a ausência de diferença entre o GT e o GP antes do tratamento também sugere que eles apresentavam o mesmo grau de dor antes do tratamento. Provavelmente, a diferença entre os resultados aqui apresentados e o de Hielm-Björkman et al. (2009) se devam ao fato de que eles utilizaram tanto animais com DCF como com osteoartrite de cotovelo. A DCF, por ser muitas vezes unilateral ou bilateral e de sinais clínicos variáveis, pode dificultar uma avaliação mais precisa por parte dos tutores.

Outra explicação para a ausência de diferença entre o carprofeno e a lactose pode se relacionar ao efeito placebo. Reportaram-se melhoras de $57 \%$ e de 40 a $45 \%$, quando os tutores e veterinários avaliaram o grau de claudicação em cães tratados com placebo, respectivamente (Conzemius e Evans, 2012). No caso em análise, a melhora foi pouco acima de $20 \%$.

Para a consistência interna, considera-se o valor de $\alpha$ de Cronbrach acima de 0,70 como aceitável (DeVellis, 2003), e o resultado aqui apresentado de 0,89 foi excelente. Quanto à repetibilidade, o intervalo de duas semanas entre o preenchimento do questionário foi aparentemente adequado, já que não houve variação nas respostas dos tutores dos cães entre os momentos basais. Tal intervalo é corroborado por Waltz et al. (2005) para o teste-reteste.

Entre as limitações inerentes a qualquer estudo clínico, foi difícil selecionar animais que se enquadrassem nos critérios de inclusão, já que, em alguns casos, havia alteração radiográfica, sem sinais de dor. Também foi difícil a adesão dos tutores e ocorreram desistências e posologia inadequada em alguns. Para cálculo do poder do teste, o tamanho da amostra se baseou nos dados de Hielm-Björkman et al. (2009), que avaliaram 17 cães por grupo. Porém, ao se realizar o cálculo, determinou-se a necessidade de se avaliarem 40 animais por grupo, e o poder foi de 0,3 , sendo que valores abaixo de 0,8 indicam que, mesmo que haja efeito no tratamento, a probabilidade de detectá-lo é muito baixa (Button et al., 2013). Outra limitação é que, como demonstrado anteriormente (Teixeira et $a l .$, 2016), a DCF não parece ser um bom modelo de dor crônica pela complexidade da claudicação.

Apesar de a EAVdor e a EAVloc apresentarem redução de seus valores ao longo do tratamento com carprofeno, tais escalas aparentemente não são indicadas para avaliar a dor, por simplificarem a experiência dolorosa, já que são unidimensionais, além de apresentarem pobre validade de conteúdo (Hielm-Bjorkman et al., 2011).

Instrumentos gerados em outra língua e cultura devem ser adaptados até se tornarem linguística e semanticamente compreensíveis para um novo idioma, porém sem perder o significado dos itens originais (Sperber, 2004). Esse processo também deve incluir uma nova avaliação das propriedades psicométricas do instrumento traduzido (Streiner e Norman, 2008).

\section{CONCLUSÕES}

$\mathrm{O}$ IDCH em português apresentou validade de constructo, critério e foi fidedigno. Pode-se, portanto, empregá-lo em países de língua portuguesa em cães com osteoartrite. A plataforma de pressão não parece ser uma ferramenta útil para avaliar o grau de claudicação na displasia coxofemoral.

\section{AGRADECIMENTOS}

À Fapesp, pelo auxílio financeiro da bolsa de pós-doutorado (2011/20619-0) e pelo auxílio científico (2010/08967-0), e à Agener União, pelo fornecimento do carprofeno. 


\section{REFERÊNCIAS}

BRASS, W. Hip dysplasia in dogs. J. Small Anim. Pract., v.30, p.166-170, 1989.

BRONDANI, J.T.; LUNA, S.P.L.; MINTO, B.W. et al. Confiabilidade e pontuação mínima relacionada à intervenção analgésica de uma escala multidimensional para avaliação de dor pós-operatória em gatos. Arq. Bras. Med. Vet. Zootec., v.65, p.153-162, 2013.

BUTTON, K.S.; IOANNIDIS, J.P.A.; MOKRYSZ, C. et al. Power failure: why small sample size undermines the reliability of neuroscience. Nat. Rev. Neurosci., v.14, p.365376, 2013.

CONZEMIUS, M.G.; EVANS, R.B. Caregiver placebo effect for dogs with lameness from osteoarthritis. J. Am. Vet. Med. Assoc., v.24, p.1314-1319, 2012.

DeVELLIS, R.F. Scale development: theory and application. 2.ed. Thousand Oaks: Sage, 2003. $205 p$.

EDAMURA, K.; KING, J.N.; SEEWALD, W. et al. Comparison of oral robenacoxibe and carprofen for the treatment of osteoarthritis in dogs: a randomized clinical trial. J. Vet. Med. Sci., v.74, p.1121-1131, 2012.

FLÜCKIGER, M. Scoring radiographs for canine hip dysplasia - the big three organisations in the world. Eur. J. Comp. Anim. Pract., v.17, p.135-140, 2007.

GUILLEMIN, F. Cross-cultural adaptation and validation of health status measures. Scand. J. Rheumatol., v.24, p.61-63, 1995.

HIELM-BJÖRKMAN, A.K.; KAPATKIN, A.S.; RITA, H.J. Reliability and validity of a visual analogue scale used by owners to measure chronic pain attributable to osteoarthritis in their dogs. Am. J. Vet. Res., v.72, p.601-607, 2011.

HIELM-BJÖRKMAN， A.K.; KUUSELA， E.; LIMAN, A. et al. Evaluation of methods for assessment of pain associated with chronic osteoarthritis in dogs. J. Am. Vet. Med. Assoc., v.222, p.1552-1558, 2003.
HIELM-BJÖRKMAN, A.K.; RITA, H.; TULAMO, R.M. Psychometric testing of the Helsinki chronic pain index by completion of a questionnaire in Finnish by owners of dogs with chronic signs of pain caused by osteoarthritis. Am. J. Vet. Res., v.70, p.727-734, 2009.

JOHNSTON, S.; McLAUGHLIN, R.; BUDSBERG, S. Nonsurgical management of osteoarthritis in dogs. Vet. Clin. N. Am. Small Anim. Pract., v.38, p.1449-1470, 2008.

LIPSCOMB, V.J.; ALIABADI, F.S.; LEES, P. et al. Clinical efficacy and pharmacokinetics of carprofen in the treatment of dogs with osteoarthritis. Vet. Rec., v.150, p.684-689, 2002.

LUST, G. An overview of the pathogenesis of canine hip dysplasia. J. Am. Vet. Med. Assoc., v.210, p.1443-1445, 1997.

MORTON, C.M.; REID, J.; SCOTT, M. et al. Application of a scaling model to establish and validate an interval level pain scale for assessment of acute pain in dogs. Am. J. Vet. Res., v.66, p.2154-2166, 2005.

OOSTERLINCK, M.; BOSMANS, T.; GASTHUYS, F. et al. Accuracy of pressure plate kinetic asymmetry indices and their correlation with visual gait assessment scores in lame and nonlame dogs. Am. J. Vet. Res., v.72, p.820-825, 2011.

REYMOND N.; SPERANZA, C.; GRUET, P. et al. Robenacoxibe vs carprofen J. Vet. Pharm. Ther., v.35, p.175-183, 2012.

RYCHEL, J.K. Diagnosis and treatment of osteoarthritis. Top. Companion Anim. Med., v.25, p.20-25, 2010.

SHARKEY, M. The challenges of assessing osteoarthritis and postoperative pain in dogs. Am. Assoc. Pharm. Sci. J., v.15, p.598-607, 2013.

SPERBER, A.D. Translation and validation of study instruments for cross-cultural research. Gastroenterology, v.126, Suppl. 1, p.124-128, 2004.

STREINER, D.L.; NORMAN G.R. Health measurement scales: a practical guide to their development and use. 4.ed., New York: Oxford University Press, 2008. 431p. 
TEIXEIRA, L.R.; LUNA, S.P.L.; MATSUBARA, L.M. et al. Owner assessment of chronic pain intensity and results of gait analysis of dogs with hip dysplasia treated with acupuncture. J. Am.Vet. Med. Assoc., v.249, p.1031-1037, 2016.

VASSEUR, P.B.; JOHNSON, A.L.; BUDSBERG, S.C. et al. Randomized, controlled trial of the efficacy of carprofen, a nonsteroidal anti-inflammatory drug, in the treatment of osteoarthritis in dogs. J. Am. Vet. Med. Assoc., v.206, p.807-811, 1995.

VOSS, K.; GALEANDRO L.; WIESTNER, T. et al. Relationships of body weight, body size, subject velocity, and vertical ground reaction forces in trotting dogs. Vet. Surg., v.39, p.863$869,2010$.
WALTON, M.B.; COWDEROY, E.; LASCELLES, D. et al. Evaluation of construct and criterion validity for the 'Liverpool Osteoarthritis in Dogs' (LOAD) clinical metrology instrument and comparison to two other instruments. PLoS One, v.8, p.e58125, 2013.

WALTZ, C.F.; STRICKLAND, O.L.; LENZ E.R. Measurement in nursing and health research. 3.ed. New York: Springer, 2005. 433p.

WISEMAN-ORR, M.L.; NOLAN, A.M.; REID, J. et al. Preliminary study on owner-reported behavior changes associated with chronic pain in dogs. Vet. Rec., v.149, p.423-424, 2001.

WISEMAN-ORR, M.L.; SCOTT, M.; REID, J. et al. Validation of a structured questionnaire as an instrument to measure chronic pain in dogs on the basis of effects on health-related quality of life. Am. J. Vet. Res., v.67, p.1826-1836, 2006. 